\title{
Influence of stack geometry on the performance of thermoacoustic refrigerator
}

\author{
B RAMESH NAYAK ${ }^{1, *}$, G PUNDARIKA ${ }^{2}$ and BHEEMSHA ARYA ${ }^{3}$ \\ ${ }^{1}$ Department of Industrial Engineering and Management, BMS College of Engineering, Bengaluru 560019, \\ India \\ ${ }^{2}$ Government Engineering College, Ramanagara 562159, India \\ ${ }^{3}$ Department of Mechanical Engineering, BMS College of Engineering, Bengaluru 560019, India \\ e-mail: b_rameshnayak@rediffmail.com
}

MS received 7 October 2015; revised 8 May 2016; accepted 12 July 2016

\begin{abstract}
The work reported in this paper is focused on the performance of a thermoacoustic refrigerator under various operating conditions. The experiments were conducted with various stack geometries fabricated with epoxy glass and Mylar material. Four stacks with different pore sizes are used to evaluate the performance of the refrigerator. Stack 1 has parallel plates of Mylar material $0.12 \mathrm{~mm}$ thick spaced $0.36 \mathrm{~mm}$ apart. Stacks 2, 3 and 4 are made of epoxy glass with pores of circular cross-section having 1, 2 and $3 \mathrm{~mm}$ diameter, respectively. The entire resonator system was constructed from aluminium material coated with polyurethane material from inside to reduce conduction heat losses. Helium gas was used as a working fluid. The experiments were conducted with different drive ratios ranging from $1.6 \%$ to $2 \%$ with varying cooling load from 2 to $10 \mathrm{~W}$. For the experiments, operating frequencies from 200 to $600 \mathrm{~Hz}$ with mean pressure varying from 2 to $10 \mathrm{bar}$ in steps of 2 bar each were considered. The temperatures of the hot end and cold end of the heat exchangers were recorded using RTDs and a data acquisition system under various operating conditions. The coefficient of performance (COP) and relative COP (COPR) are evaluated. Results show that COP of the refrigerator rises with increase of cooling load and decreases at higher drive ratio. It was also observed that the temperature difference between the hot end and cold end of the stack is higher at $2 \mathrm{~W}$ cooling load for $400 \mathrm{~Hz}$ operating frequency. The temperature difference between the hot end and cold end of the stack was observed to be $19.4,17.2,14$ and $12.4^{\circ} \mathrm{C}$ for stacks 1, 2, 3 and 4, respectively, for 10 bar mean pressure and $2 \mathrm{~W}$ cooling load. The temperature difference and COP of the parallel plate stack are better compared with other stack geometries.
\end{abstract}

Keywords. COP; COPR; cooling load; drive ratio; frequency; stack geometry.

\section{Introduction}

The discovery of thermoacoustic phenomenon goes back to more than a century ago, but significant work in this area was started about two decades ago at the Los Alamos National Laboratory by the research group of Greg Swift. They developed different types of thermoacoustic refrigerators and heat engines [1]. A few other research groups are also working in this area. However, the development of such devices is still at preliminary stages.

The interaction of sound and heat was recognized over 200 years ago in the disagreement between Newton and Laplace on whether propagation of sound in air is adiabatic or isothermal (it is largely an adiabatic process-one of the few times that Newton was wrong). Glass blowers in the 19th century noticed that freshly blown hot glass bulbs that were attached to cool stems would occasionally sing. This

*For correspondence effect was explained by Sondhauss, who qualitatively suggested that there existed a relationship between the pitch of the sound and the geometry of the bulbs and stems. Sondhauss made a note in his work of "Glowing glass harmonica" that preceded his investigation by 40 years.

In 1896, Lord Rayleigh explained the Sondhausss effect, and correctly understood the natural phasing of the acoustic motion, pressure and temperature changes with the conductive heat transfer that could cause an acoustic oscillation to be sustained. It was not until Nicholas Rott and his collaborators worked out a solid quantitative understating of thermoacoustics that acousticians were able to create intelligent thermoacoustic engine designs. A more complete history, with many references, can be found in Swift's excellent review of thermoacoustics published in 1988 [2].

Rott [3] developed a theoretical and analytical foundation for thermoacoustic heat pumps and refrigerators. Merkli et al [4, 5] and Hofler [6] helped initiate attempts at the development of thermoacoustic devices intended for 
practical cooling applications. Herman and Wetzel [7] studied design optimization of thermoacoustic refrigerators using the short-stack boundary layer approximation and Minner et al [8] reported that the choice of working fluid and heat exchanger configuration were critical design factors affecting the performance of the thermoacoustic system. Knio [9-11] performed numerical studies on a thermoacoustic refrigeration system with emphasis on thermally stratified flow in the neighbourhood of an idealized thermoacoustic stack. Energy flux density around the heat exchanger was visualized and implications on the heat exchanger design were examined. Belcher et al [12] studied working gases suitable for use in thermoacoustic systems.

Tijani et al [13] designed, constructed and tested a thermoacoustic refrigerator for cooling power of $4 \mathrm{~W}$. The COP of 1.06 is reported for the lowest temperature of $-65^{\circ} \mathrm{C}$. Garrett and Poese [14] measured the performance of a thermoacoustic refrigerator driven at relatively high amplitudes. Garret et al [15] developed a new spacecraft cryocooler, which used resonant high-amplitude sound waves in inert gases to pump heat. This cryocooler was used in the space shuttle Discovery. Tijani et al [16] achieved temperature as low as $-65^{\circ} \mathrm{C}$ in their device. They used it to study the effect of some important thermoacoustic parameters, such as the Prandtl number and the stack plate spacing. Bailliet et al [17] measured the acoustic power in the resonator of a thermoacoustic refrigerator by laser Doppler anemometry [LDA] together with microphone acoustic pressure measurement. They found good agreement between the experimental and theoretical results. Akhavanbazaz et al [18] investigated the impact of gas blockage on the performance of thermoacoustic refrigerator for three cases, that is, no heat exchanger, heat exchanger with a small thermal contact area and heat exchanger with large thermal contact area. The results show that the blockage has a significant impact on thermoacoustic process inside the stack, and also suggest that the optimization of these parameters is necessary in order to optimize the performance of thermoacoustic refrigerator.

Nsofor et al [19] studied the performance of the thermoacoustic refrigerating system with respect to some critical operating parameters. The temperature difference between the hot end and the cold end of the stack ranged from 0 to $15^{\circ} \mathrm{C}$.

Tasnim et al [20] numerically investigated the effects of variation in working fluids and operating conditions on the performance of a thermoacoustic refrigerator. The performance of a thermoacoustic refrigerator is evaluated based on the cooling power, coefficient of performance (COP) and the entropy generation rate within the device. The effect of the variation of the working fluid is observed by changing the Prandtl number $(\operatorname{Pr})$ between 0.7 and 0.28 . The operating conditions investigated are drive ratio (DR), stack plate spacing $\left(y_{0}\right)$ and mean pressure $\left(p_{\mathrm{m}}\right)$. Coefficient of performance increases $78 \%$ by reducing the Prandtl number from 0.7 to 0.28 at stack plate spacing equal to 3.33 times the thermal penetration depth for atmospheric pressure and a drive ratio of $1.7 \%$.

Bheemsha et al [21] designed and fabricated a cooling power of $10 \mathrm{~W}$ for both air and helium as the working medium. The stack COP was optimized and found to be 2.5. The acoustic losses in the small diameter resonator tube were minimized by taking the optimized value of the diameter ratio (D2/D1) equal to 0.43 .

\section{Experimental set-up}

A thermoacoustic refrigerator is designed for a cooling power of $10 \mathrm{~W}$ considering pure helium and compressed air as the working media. The design of various components such as stacks, different stack geometries, variable pressure, resonator tube, heat exchangers and buffer volume has been carried out with the help of relevant thermoacoustic equations [2].

Figure $1 \mathrm{a}$ and $\mathrm{b}$ shows a schematic layout and experimental setup of the thermoacoustic refrigerator constructed at the thermoacoustic laboratory of BMS College of Engineering, Bengaluru, India. The principal parts of thermoacoustic refrigerator consists of an acoustic driver, two heat exchangers, a stack that acts as a heat pumping element and the resonator system.

The thermoacoustic refrigerator is designed and optimized for a quarter wave length $(\lambda / 4)$ resonator. A cone shape buffer volume is accommodated in order to simulate open end condition. The resonator system consists of a large diameter tube $\mathrm{D}_{1}(70.06 \mathrm{~mm})$ and small diameter tube $\mathrm{D}_{2}(30.12 \mathrm{~mm})$; the ratio of small diameter to large diameter was restricted to 0.43 to minimize energy losses. The entire resonator system was constructed from aluminium. The inner surface of the resonator tube was coated with polyurethane material to reduce conduction heat loss. Figure 2 shows the different geometries of the stacks used in the experimental setup. Stack 1 is of $40.55 \mathrm{~mm}$ length and $69 \mathrm{~mm}$ diameter having parallel plates constructed of 0.12 $\mathrm{mm}$ thick Mylar sheets spaced $0.36 \mathrm{~mm}$ apart. The stack plate holder was manufactured using wire EDM. Stacks 2, 3 and 4 , made of epoxy glass with pores of circular crosssection having 1, 2 and $3 \mathrm{~mm}$ diameter, respectively, were manufactured using CNC. These stacks are not designed and optimized based on linear thermoacoustic theory unlike stack 1 . They are fabricated to a length of $40.55 \mathrm{~mm}$ and outer diameter of $69 \mathrm{~mm}$ in order to compare the results with stack 1. Copper-tube-type heat exchangers were employed at two ends of the stacks. A $10 \mathrm{~W}$ electric heater is placed on a cold heat exchanger of $1.9 \mathrm{~mm}$ diameter and $498.6 \mathrm{~mm}$ length, for cooling power measurement. The hot heat exchanger of diameter $3.8 \mathrm{~mm}$ and $249.3 \mathrm{~mm}$ length is provided with cooling arrangement. An acoustic driver with a variable power input of $0-120 \mathrm{~W}, 8 \Omega$ was selected in conjunction with an amplifier and audio generator to produce required frequency $(100-600 \mathrm{~Hz})$ sound waves in the 

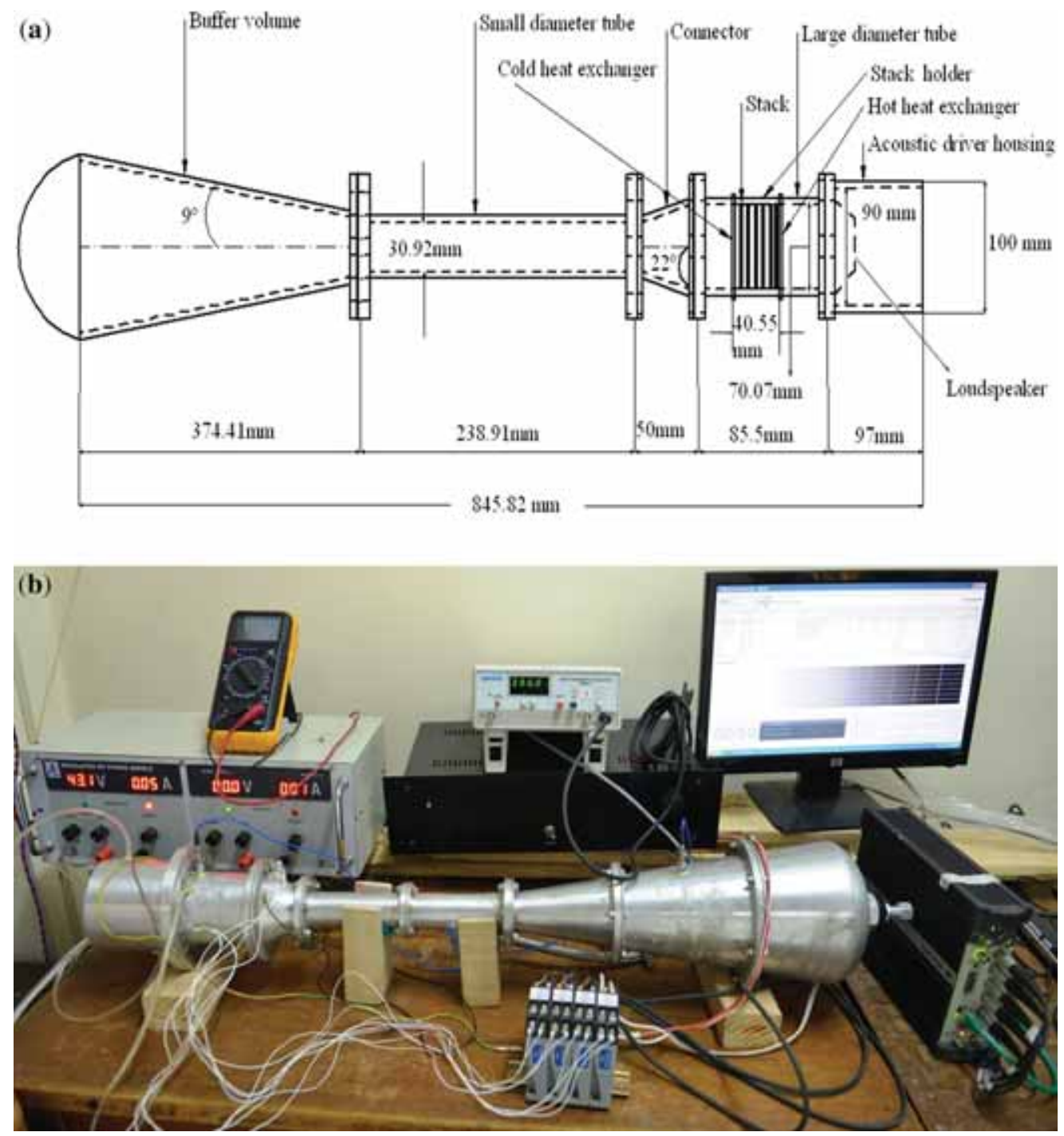

Figure 1. (a) Schematic diagram of a standing-wave thermoacoustic refrigerator. (b) Experimental set-up of the thermoacoustic refrigerator.

resonator. The acoustic driver was provided with cooling arrangement to take care of the heat generation inside the sealed casing. Two pressure transducers PCB Piezotronics 101A06 SN 6801 and 6802 (voltage sensitivity $146.4 \mathrm{mV} /$ bar) are placed, one near the driver end and the other one as buffer in the range 1-20 bar, which were used to measure the dynamic pressure and resonance frequency. Pressure transducers were mounted with the help of threading and O-ring sealing. Disc type Heatcon RTD PT 100 Sensors of diameter $15 \mathrm{~mm}$ and thickness $2 \mathrm{~mm}$ with $2 \mathrm{~m} 3 \mathrm{KVA}$ insulation cable operating in the temperature range -25 to $100^{\circ} \mathrm{C}$ were installed on the hot and cold heat exchangers to measure the temperature distribution with an 8-channel PULSE Type 3560-B-130 data acquisition unit of Bruel and Kjear with multi-channel analog input operating in the frequency range $0 \mathrm{~Hz}-25.6 \mathrm{kHz}$. The amplifier is used to amplify the output signal of the function generator SM 5077 (Scientific). The pressure transducer and RTD were calibrated with suitable calibrators from the suppliers. The entire thermoacoustic system was made leak proof and tested for a pressure up to 20 bar (figure 1b).

\section{Experimental procedure}

Experiments were conducted to evaluate the performance of the thermoacoustic refrigeration system under various operating conditions with different stack geometries. The resonator tube was filled with helium gas with the help of regulating systems. The initial mean pressure was varied from 2 to 10 bar in steps of 2 bar. The desired frequency was set and then increased slowly from 200 to $600 \mathrm{~Hz}$ in steps of $100 \mathrm{~Hz}$ for each trial. The cooling load was controlled using a resistance heating coil placed on the cold heat exchanger. The experimental data were recorded for each trial after the system was stabilized. The experiments 

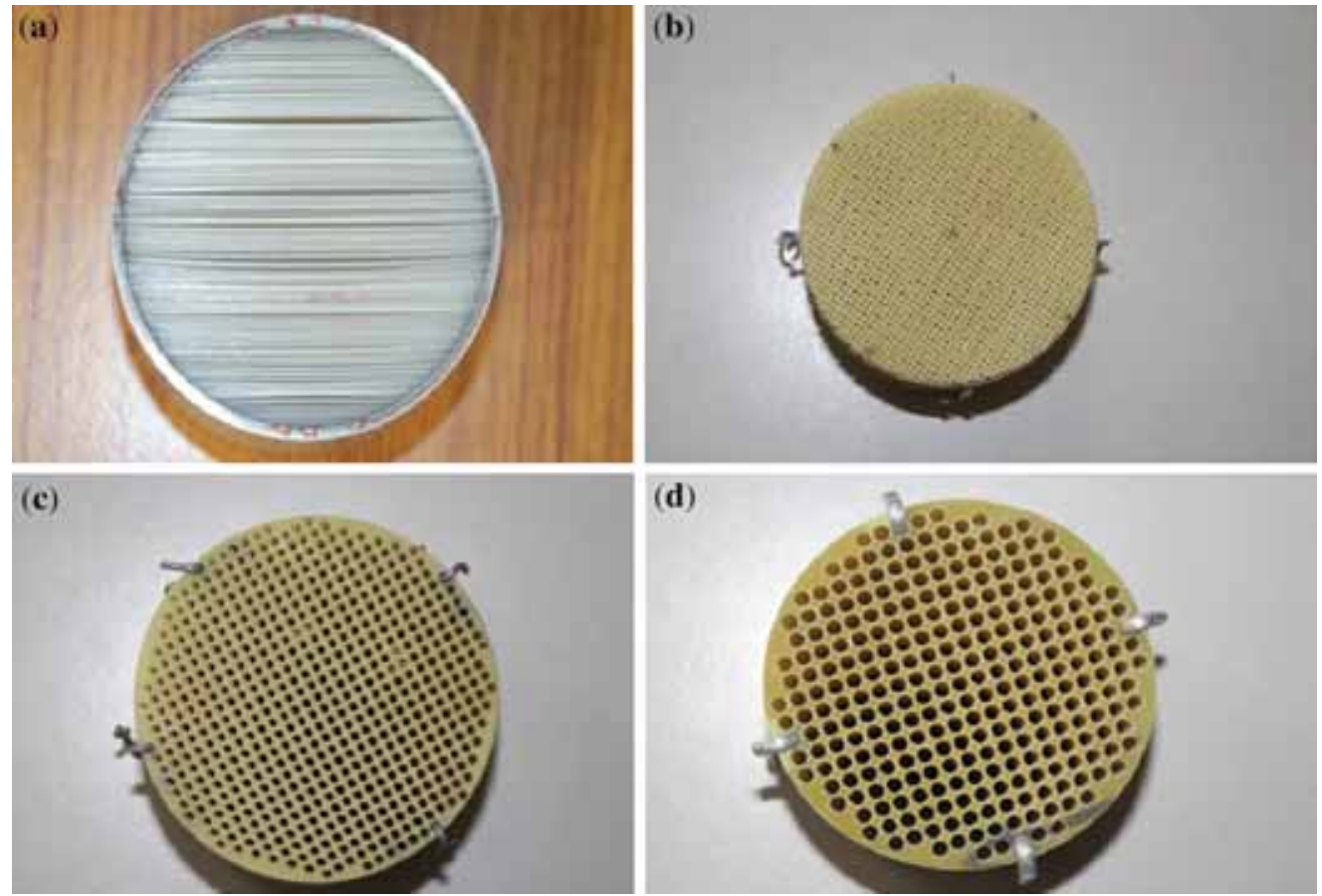

Figure 2. (a) Stack 1 (Parallel plates). (b) Stack 2 (1 mm dia). (c) Stack 3 (2 mm dia). (d) Stack 4 (3 mm dia).
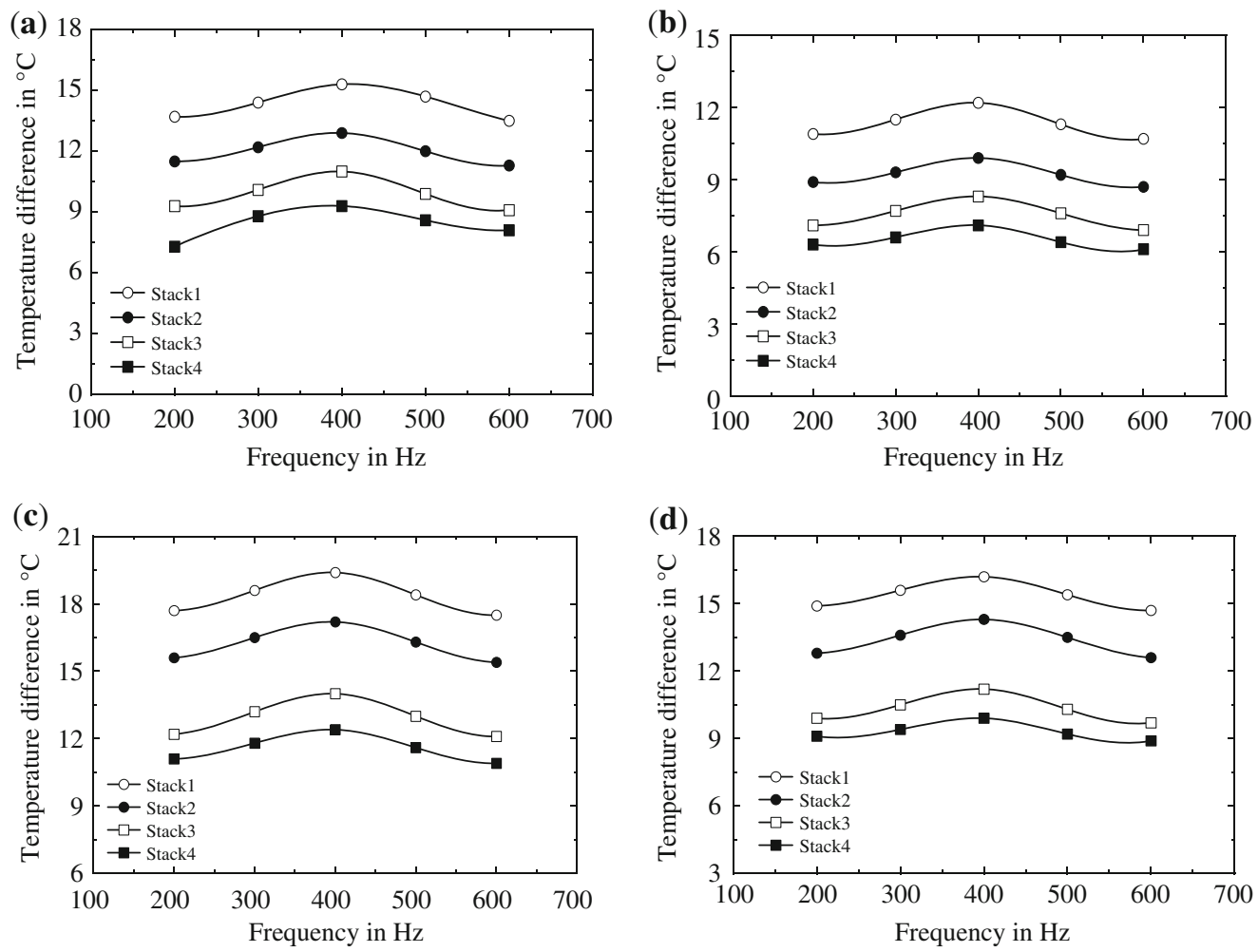

Figure 3. (a) Variation of $\Delta T$ with frequency for different stack geometries at 2 bar mean pressure and $2 \mathrm{~W}$ cooling load at DR $2 \%$. (b) Variation of $\Delta T$ with frequency for different stack geometries at 2 bar pressure and $10 \mathrm{~W}$ cooling load at DR $2 \%$. (c) Variation of $\Delta T$ with frequency for different stack geometries at 10 bar mean pressure and $2 \mathrm{~W}$ cooling load at DR $2 \%$. (d) Variation of $\Delta T$ with frequency for different stack geometries at 10 bar pressure and $10 \mathrm{~W}$ cooling load at DR $2 \%$. 

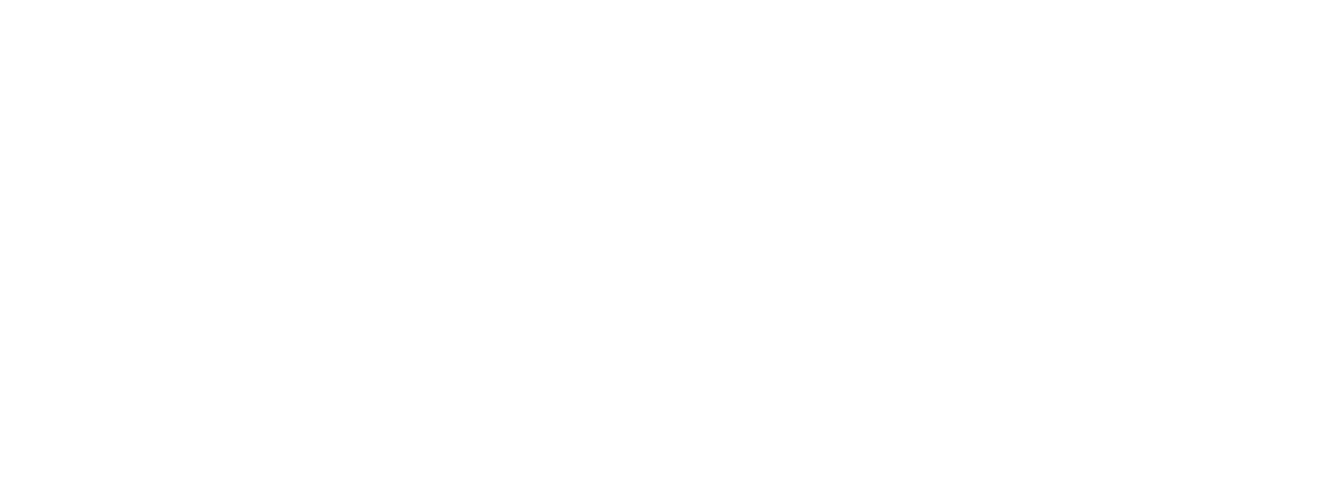

Figure 4. (a) Variation of $\Delta T$ with cooling load different stack geometries at 2 bar mean pressure, DR $2 \%$ and $400 \mathrm{~Hz}$ frequency mean. (b) Variation of $\Delta T$ with cooling for load for different stack geometries at 10 bar pressure, DR $2 \%$ and $400 \mathrm{~Hz}$. frequency.

were conducted by varying frequency, cooling load and mean pressure for each set of experiments. The experiments were repeated for different input powers to the acoustic driver, thereby maintaining the desired drive ratio. Cooling arrangements for the acoustic driver and hot heat exchanger were made to remove heat generated.

\section{Results and discussion}

The thermal performance of a thermoacoustic refrigerator subjected to various operating conditions such as frequency, mean pressure, stack geometry, acoustic input power and cooling load are discussed in this section.

The performance of the thermoacoustic refrigerator is evaluated using performance parameters such as the cooling power, acoustic input power and temperature difference for various operating conditions. The electric power introduced into the system is converted into work, i.e. acoustic power in the acoustic driver. The electro-acoustic conversion efficiency for commercial loudspeakers is on the order of $3 \%$. However in the present work the driver efficiency of $16 \%$ is considered as recommended by the manufacturer and sufficient care was taken to verify the efficiency of the driver using a calibration chart. The coefficient of performance (COP) is the ratio of cooling load to the acoustic input power. The Carnot COP $\left(\mathrm{COP}_{\mathrm{C}}\right)$ is calculated as the ratio of cold end temperature to the temperature difference of hot and cold ends, i.e., $T_{\mathrm{C}} /\left(T_{\mathrm{H}}-\right.$ $T_{\mathrm{C}}$ ). COPR is the ratio of the actual COP to $\mathrm{COP}_{\mathrm{C}}$ and is often used to quantify the performance of refrigerators.

\subsection{Effect of frequency}

The variation of temperature difference $(\Delta T)$ with frequencies at constant mean pressure and cooling load is shown in figure $3 \mathrm{a}-\mathrm{d}$, illustrating the effect of frequency on the temperature. It is evident from the figures that the temperature difference across the stack rises with increase in frequency. At about $400 \mathrm{~Hz}$ the difference in temperature reaches the maximum value. This variation is due to the presence of sound energy, making the resonator tube to vibrate. This clearly indicates the relation between operating frequency and natural resonance frequency of the refrigerator, which is responsible for the change in temperature difference across the hot and cold ends of the stack.

It is also noticed that with a further increase in operating frequency beyond $400 \mathrm{~Hz}$ the temperature difference keeps reducing. This is due to the presence of weak thermal
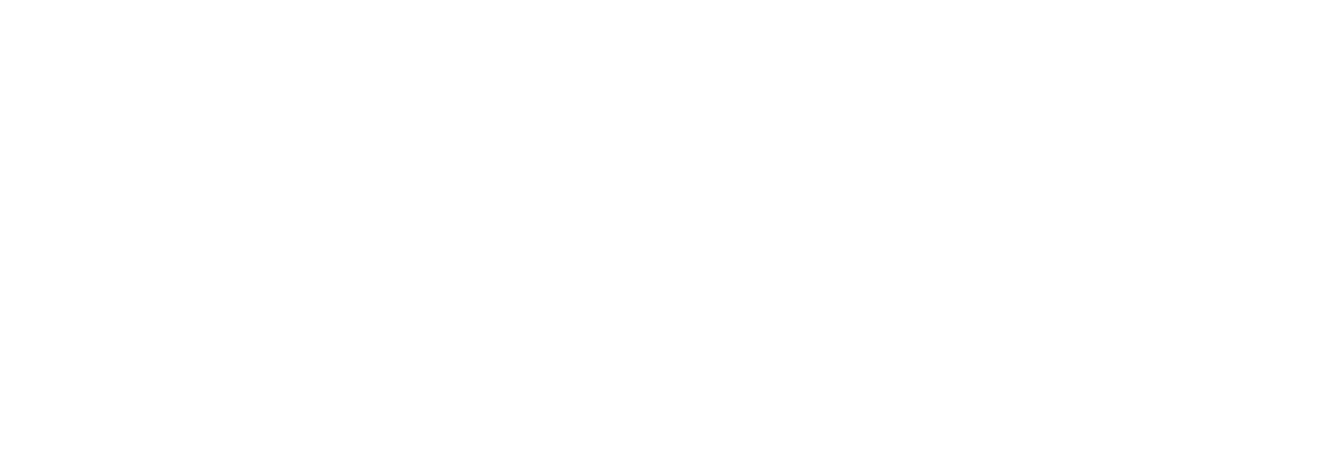

Figure 5. (a) Variation of COP with Cooling Load stack1 at different Drive Ratio. (b) Variation of COP with for Cooling Load for different stacks at Drive Ratio $1.6 \%$. 

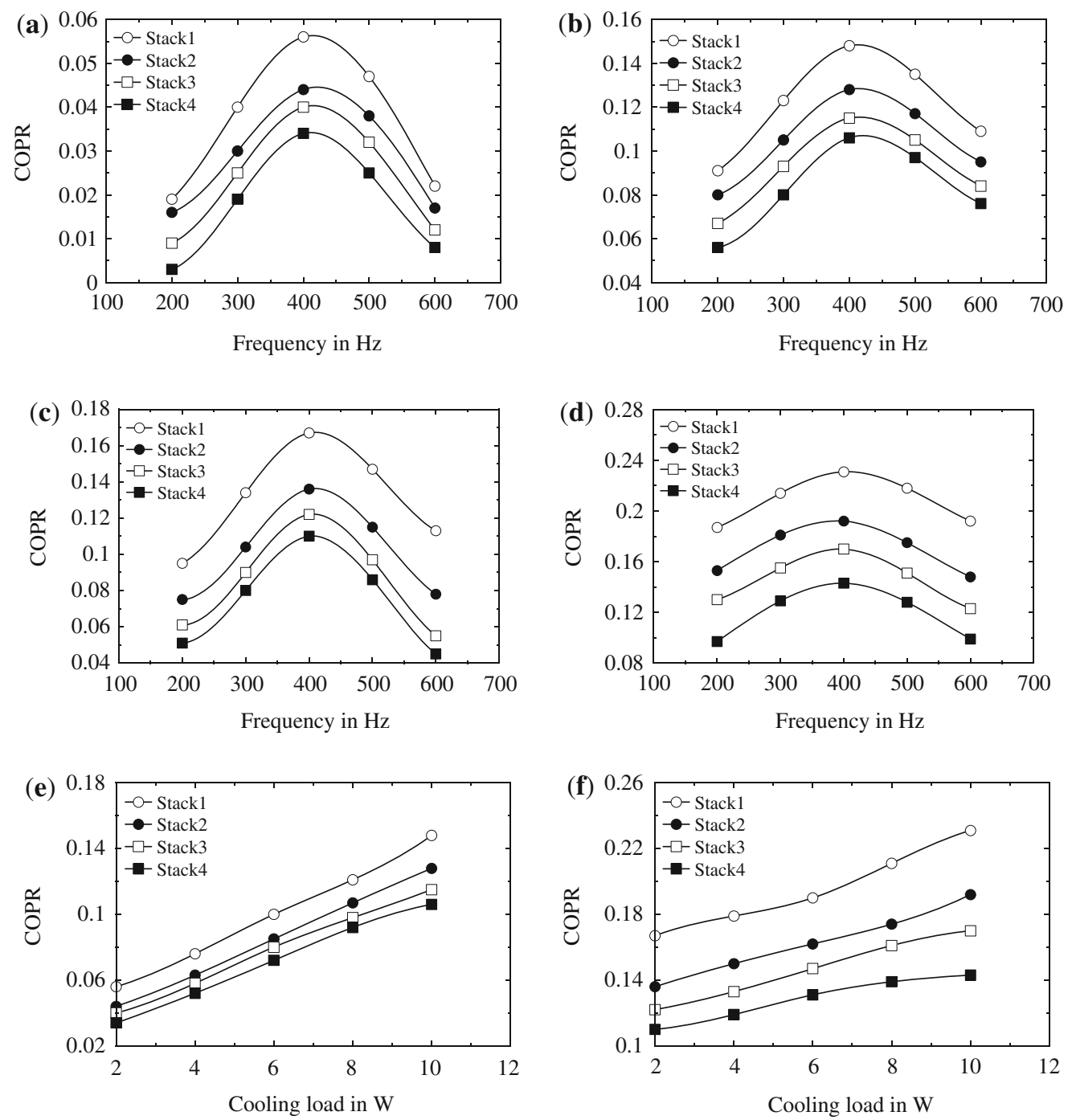

Figure 6. (a) Variation of COPR with Frequency for different stack geometry at 2 bar mean pressure and $2 \mathrm{~W}$ Cooling Load. (b) Variation of COPR with Frequency for different stack geometry at 2 bar mean pressure and10W Cooling Load. (c) Variation of COPR with Frequency for different stack geometry at 10 bar mean pressure and 2W Cooling Load. (d) Variation of COPR with Frequency for different stack geometry at 10 mean pressure and10W Cooling Load. (e) Variation of COPR with Cooling Load for different stack geometry at 2 bar mean pressure, Drive Ratio $1.6 \%$ and $400 \mathrm{~Hz}$ frequency. (f) Variation of COPR with Cooling Load for different stack geometry at 10 bar mean pressure, Drive Ratio $1.6 \%$ and $400 \mathrm{~Hz}$ frequency.

penetration depth in the stack at higher operating frequencies, leading to the increase in viscous loss and reduction of the thermoacoustic effect.

The magnitude of the temperature difference between the hot end and the cold end of the stack rises with increase in mean pressure for all the stacks and the maximum temperature difference was found for a cooling load of $2 \mathrm{~W}$ corresponding to a mean pressure of 10 bar.

\subsection{Effect of cooling load}

Figure $4 \mathrm{a}$ and $\mathrm{b}$ presents the variation of temperature difference with cooling load for constant mean pressure and frequency. Temperature difference is a function of the cooling load. Higher temperatures are attained with higher dynamic pressure. Measurements are made using three drive ratios for different stack geometries with helium as the working medium. The figures show that the temperature difference is maximum corresponding to a cooling load of 2 $\mathrm{W}$. This variation is due to the fact that the acoustic power input available is sufficient to remove the heat generated at cold end of the stack, and for higher cooling loads it is not sufficient for all pressures due to the very low electroacoustic energy conversion efficiency of the acoustic driver. It is also evident from the figures that the temperature difference between hot end and cold end for stack 1 is found to be more compared with other geometries for the same operating conditions. 
(a)
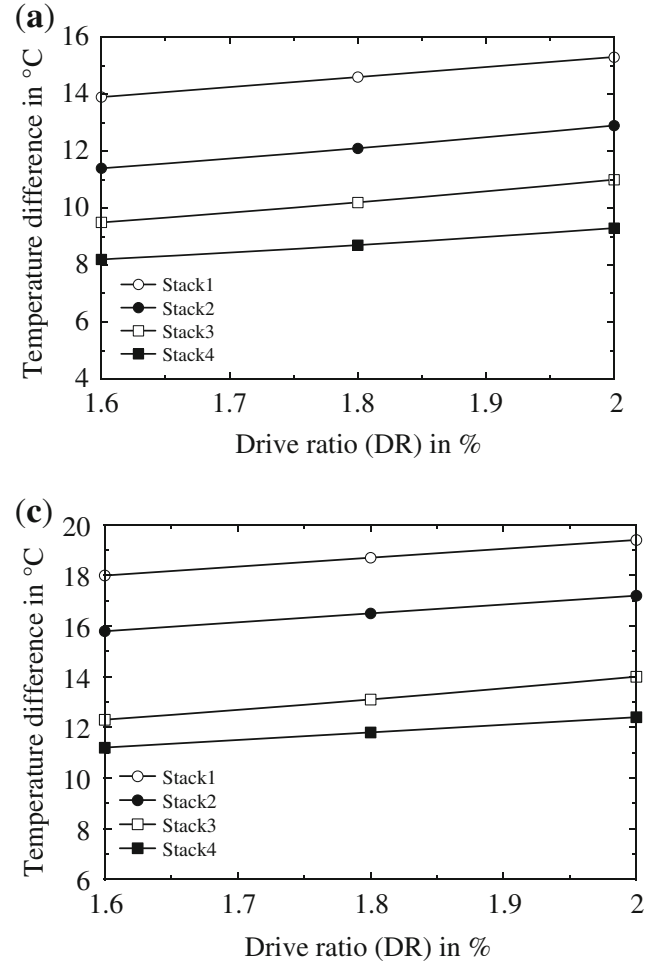
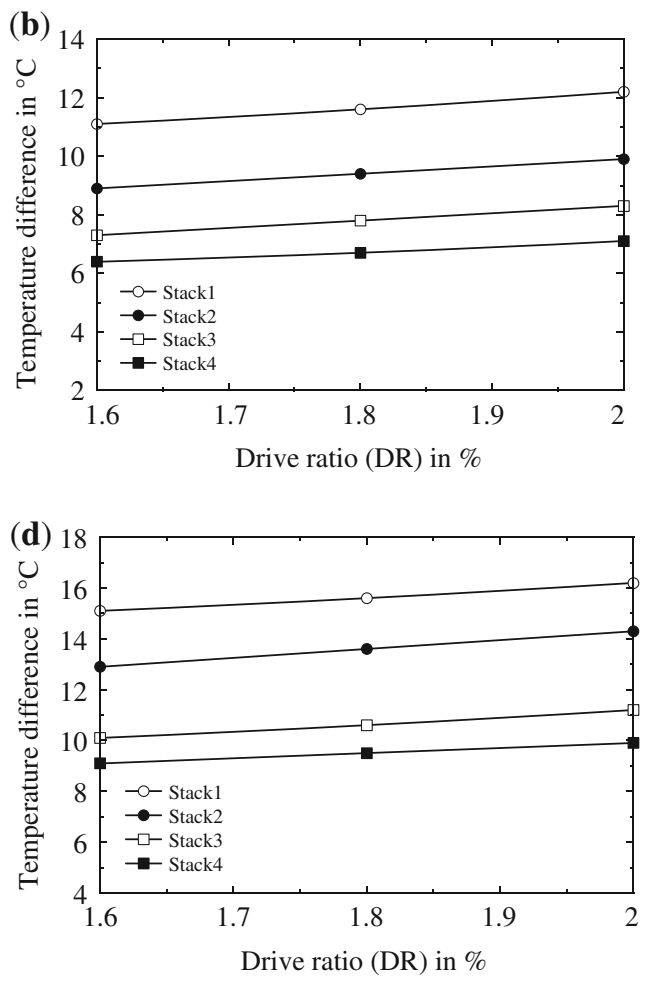

Figure 7. (a) Variation of $\Delta T$ with Drive Ratio for different stack geometry at 2 bar mean pressure and $2 \mathrm{~W}$ Cooling Load. (b) Variation of $\Delta T$ with Drive Ratio for different stack geometry at 2 bar mean pressure and 10W Cooling Load. (c) Variation of $\Delta T$ with Drive Ratio for different stack geometry at 10 bar mean pressure and $2 \mathrm{~W}$ Cooling Load. (d) Variation of $\Delta T$ with Drive Ratio for different stack geometry at 10 bar mean pressure and 10W Cooling Load.

\subsection{Variation of $C O P$}

Figure 5a shows the variation of COP with cooling load for different drive ratios with respect to stack 1 . COP rises with the increase in cooling load as it is directly proportional to cooling load. It is found that COP increases with decrease in drive ratio due to increase in acoustic power input. Similar variations were observed for other stack geometries and hence they are shown in figure $5 \mathrm{~b}$. Stack 1 is found to be more efficient compared with other geometries because of its effective thermal penetration depth, leading to efficient heat removal from the cold end of the stack.

\subsection{Variation of COPR}

Figure $6 a-d$ shows the variations of COPR with frequency for different stack geometries shown therein for helium as the working fluid. It can be seen from these figures that COPR is the maximum for stack 1 compared with other stacks. This phenomenon shows the importance of the stack spacing and thermal and viscous penetration depth on the performance of the thermoacoustic refrigerator. COPR rises with the increase in COP as it is proportional to the COP; it is found to be maximum at $400 \mathrm{~Hz}$ frequency corresponding to a mean pressure of 10 bar and $10 \mathrm{~W}$ cooling load for stack 1 at drive ratio $1.6 \%$. This is due to the presence of weak thermal penetration depth in the stack at higher operating frequencies, leading to the increase in viscous loss and reduction of the thermoacoustic effect.

The COPR also rises with increase in cooling load as it is directly proportional to the $\mathrm{COP}$ as shown in figure 6e and $\mathrm{f}$ for mean pressure of 2 bar and 10 bar, respectively. The COPR increases with the mean pressure and approaches its maximum limit as it is proportional to thermoacoustic heat pumping power. Hence, the effect of mean pressure and other operating conditions could be different for systems operating with high drive ratios. Small energy losses may take place due to turbulence and flow discontinuities in the resonator cross-sectional area, particularly in high-velocity regions.

\subsection{Effect of drive ratio}

The temperature difference between hot and cold ends of the stack rises with the increase in drive ratio for all stack geometries at constant cooling load and mean pressure as shown in figure $7 \mathrm{a}-\mathrm{d}$. Results indicate that stack 1 is more capable of creating large temperature difference compared 
with other stack geometries. This variation is due to better thermal and viscous penetration depth as the spacing between the two plates in stack 1 is quite small. The drive ratio plays a very important role in enhancing thermoacoustic effect, leading to better heat transfer and hence temperature difference.

\section{Conclusions}

An experimental study has been carried out in order to investigate the influence of a few basic thermoacoustic parameters on the behaviour of thermoacoustic refrigerator under various operating conditions.

The influence of stack geometry on the performance of the refrigerator is studied. The experimental results indicate that stack 1 performs better in terms of cooling power.

The maximum temperature difference of about 19.4, $17.2,14$ and $12.4^{\circ} \mathrm{C}$ was achieved, respectively, with stacks $1,2,3$ and 4 when helium was used as the working fluid for 10 bar mean pressure corresponding to a cooling load of 2 $\mathrm{W}$ at $400 \mathrm{~Hz}$ frequency with drive ratio of $2 \%$.

The highest COPs of $1.715,1.423,1.184$ and 1.055 were achieved with stacks $1,2,3$ and 4 , respectively, when helium was used as the working fluid for 10 bar mean pressure corresponding to a cooling load of $10 \mathrm{~W}$ at $400 \mathrm{~Hz}$ frequency with drive ratio of $1.6 \%$. The temperature difference and COP of the parallel plate stack are better compared with other stack geometries.

\section{References}

[1] Swift S W 1995 Thermoacoustic engines and refrigerators. Phys. Today 48(7): 22-28

[2] Swift G W 1988 Thermoacoustic engines. J. Acoust. Soc. Am. 84(4): 1145-1180

[3] Rott N 1980 Thermoacoustics. Adv. Appl. Mech. 20: 135-175

[4] Merkli P and Thomann H 1975 Thermoacoustic effects in a resonance tube. J. Fluid Mech. 70: 1611

[5] Merkli P and Thomann H 1975 Transition to turbulence in oscillating pipe flow. J. Fluid Mech. 68: 567-575

[6] Hofler T J 1986 Thermoacoustic refrigerator design and performance, $\mathrm{PhD}$. Dissertation, Physics Department, University of California at San Diego, CA
[7] Herman C and Wetzel M 1997 Design and optimization of thermoacoustic refrigerators. Int. J. Refrig. 20(1): 3-21

[8] Minner B L, Braun J E and Mongeau L 1997 Theoretical evaluation for the optimal performance of a thermoacoustic refrigerator. ASHRAE Transactions Proceedings of the Winter Meeting, Atlanta, GA, 103(1), pp 873-887

[9] Knio O M and Worlikar A S 1996 Numerical simulation of a thermoacoustic refrigerator I-unsteady adiabatic flow around the stack. J. Comput. Phys. 127: 424-451

[10] Knio O M 1998 Numerical simulation of a thermoacoustic refrigerator II-stratified flow around the stack. J. Comput. Phys. 144(2): 299-324

[11] Knio O M and Worlikar A S 1999 Numerical study of oscillatory flow and heat transfer in a loaded thermoacoustic stack. Numer. Heat Transfer part-A: Appl. 35(1): 49-65

[12] Belcher J R, William V Slaton, Richard Raspet, Henry E Bass and Jay Lightfoot 1999 Working gases in thermoacoustic engines. J. Acoust. Soc. Am. 105(5): 2677-2684

[13] Tijani M E H, Zeegers J C H and de Waele A T A M 2002 Design of thermoacoustic refrigerators. J. Cryogenics 42 : 49-57

[14] Garrett S L and Poese M E 2000 Performance measurements on a thermoacoustic refrigerators driven at high amplitudes. J. Acoust. Soc. Am. 107(5): 2480-2486

[15] Garrett S L, Hofler T J and David K Perkins 1993 Thermoacoustic refrigerators for space applications, J. Thermo Phys. Heat Transfer 7(4): 595-599

[16] Tijani M E H, Zeegers J C H, and de Waele A T A M 2002 Construction and performance of a thermoacoustic refrigerator. Cryogenics 42: 59-66

[17] Bailliet H, Lotton P, Bruneau M and Gusev V 2000 Acoustic power flow measurement in a thermoacoustic resonator by means of Laser Dropper Anemometry (LDA) and micro phonic measurement. J. Appl. Acoust. 60: 1-11

[18] Masoud Akhavanbazaz, Kamran Siddiqui M H and Rama Bhat B 2007 The impact of gas blockage on the performance of a thermoacoustic refrigerator. J. Exper. Therm. Fluid Sci. 32: 231-239

[19] Emmanuel C Nsofor and Azrai Ali 2009 Experimental study on the performance of the thermoacoustic refrigerating system. Appl. Therm. Eng. 29: 2672-2679

[20] Tasnim S H, Mahmud S and Fraser R A 2012 Effects of variation in working fluids and operating conditions on the performance of a thermoacoustic refrigerator. Int. Commun. Heat Mass Transfer 39: 762-768

[21] Bheemsha, Ramesh Nayak B and Pundarika G 2011 Design and optimization of a thermo acoustic refrigerator. Int. J. Emerging Trends Eng. Develop. 2(1): 47-65 